\title{
Evaluación de portainjertos criollos de Lagenaria siceraria en la producción de sandía injertada
}

\author{
Evaluation of creoles rootstocks Lagenaria siceraria on yield \\ in grafted watermelon
}

\author{
Ángel Manuel Suárez-Hernández, Onécimo Grimaldo-Juárez*1, Alejandro Manelik García-López \\ Daniel González-Mendoza1, María Victoria Huitrón-Ramírez ${ }^{2}$
}

\begin{abstract}
RESUMEN
El injerto en hortalizas es una alternativa en los sistemas de producción de cultivos bajo condiciones adversas, siendo de mayor importancia portainjertos de Cucurbita y Lagenaria en el cultivo de sandía. La especie Lagenaria siceraria se encuentra distribuida en México; sin embargo, el uso de materiales criollos como portainjerto es limitado o nulo, por lo que, la presente investigación tuvo como objetivo evaluar y seleccionar las mejores combinaciones de portainjertos criollos de L. siceraria en dos variedades de sandía. El experimento se realizó en un arreglo factorial $2 \times 7$ dentro de un diseño completamente al azar y tres repeticiones por tratamiento. Las variables evaluadas fueron producción precoz, rendimiento total, firmeza de pulpa, proporción de pulpa en fruto y contenido de sólidos solubles. La mayor precocidad de sandía se obtuvo con la variedad 2800 superando en 12,35\% a Sangría, y en el caso de portainjertos, la tendencia generalizada fue mayor precocidad con respecto a las plantas control. El rendimiento se favoreció por el injerto principalmente con las combinaciones de L43 y L56. La proporción de pulpa fue superior en 4,58\% en la variedad 2800 con respecto a Sangría, mientras que, no se presentaron efectos significativos por la combinación de injerto. El contenido de ${ }^{\circ}$ Brix fue un parámetro con efectos significativos en la interacción de variedad por combinación de injerto, registrándose mayores incrementos en esta variable en las combinaciones de los portainjertos con la variedad Sangría. Los portainjertos con mayor efecto en precocidad, rendimiento y calidad de fruto fueron L43 y L50, los que pueden ser considerados para programas de mejoramiento posteriores.
\end{abstract}

Palabras clave: sandía, Lagenaria siceraria, injerto, portainjertos criollos.

\begin{abstract}
Vegetable grafting is an alternative in crop production systems under adverse conditions. The most important rootstocks are Cucurbita and Lagenaria in the cultivation of watermelon. The Lagenaria siceraria specie is distributed in Mexico, however, the use of natives materials as rootstock is limited or non-existent, so that this investigation was to evaluate and select the best combinations of native rootstock $\mathrm{L}$. siceraria and two watermelon varieties. The experimental design utilized was completely randomized with factorial arrangement $2 x 7$ and three replicates per treatment. The variables evaluated were early production, total yield, pulp firmness, pulp proportion of fruit and total soluble solids. The production precocity was greatest with variety 2800 exceeding by $12.35 \%$ to Sangría, and in the case of rootstocks, the general trend was higher precocity with respect to control plants. The yield was favored by graft mainly combinations L43 and L56. The proportion of pulp was higher in 2800 compared with Sangria, whereas no significant effects observed by combination of graft. The total soluble solids was a parameter with significant effects on the interaction between variety and combining graft, registering higher increases in this variable in combinations of rootstocks with variety Sangría. The rootstocks more impact on precocity, yield and fruit quality were L43 and L50, which can be considered for subsequent improvement programs.
\end{abstract}

Key words: watermelon, Lagenaria siceraria, grafting, creoles rootstocks.

1 Instituto de Ciencias Agrícolas, Universidad Autónoma de Baja California (ICA-UABC). Carretera a Delta s/n, Ejido Nuevo León, Baja California, México.

2 Instituto Tecnológico de Colima. Av. Tecnológico 1, Villa de Álvarez, 28976, Colima, Col. México.

* Autor por correspondencia: onecimo.grimaldo@uabc.edu.mx

Fecha de Recepción: 11 Marzo, 2016.

Fecha de Aceptación: 10 Enero, 2017.

DOI: $10.4067 / \mathrm{S} 0718-34292017005000002$ 


\section{Introducción}

El injerto en hortalizas es una alternativa ecológica para el control de enfermedades del suelo en la producción de cultivos de solanáceas y cucurbitáceas (Crino et al., 2007; Barrett et al., 2012), además de inducir mayor tolerancia a condiciones de salinidad de suelos, sequía, altas y bajas temperaturas (Colla et al., 2006; Schwarz et al., 2010). Los efectos en las plantas son expresados en mayor crecimiento y producción con respecto a las plantas sin injerto (Tarchoun et al., 2005).

En el cultivo de sandía, las plantas comúnmente son injertadas en portainjertos de Cucurbita maxima, C. moschata, híbridos de cucúrbita (C. maxima $\mathrm{x}$ C. moschata), y Lagenaria siceraria (Lee et al., 2010) y la respuesta generalizada ha sido mantener o incrementar el rendimiento (Alan et al., 2007); sin embargo, los días a cosecha al igual que los parámetros de calidad de los frutos son modificados por la combinación portainjertovariedad (Petropoulos et al., 2014; Soteriou et al., 2014). Los cambios inducidos en las plantas injertadas con portainjertos de L. siceraria es necesario conocerlos previamente antes de emplearse de forma comercial.

L. siceraria es considerada una especie originaria de África; sin embargo, se tiene evidencia que en América fue una de las primeras especies cultivadas en los años 8000 a 10000 a.C. (Richardson, 1972). En México, actualmente L. siceraria se distribuye en varios estados de la república mexicana, donde su cultivo se reduce a huertos familiares con interés ornamental, además de emplear sus frutos como recipientes para el almacenamiento de agua o bien como artesanía decorada con diferentes colores y figuras (Lira y Rodríguez, 2006; Chimonyo y Modi, 2013) . El conocimiento como portainjerto ha sido escaso, a pesar de la evidencia de las múltiples ventajas de dicha especie en la producción de sandía. Por lo anterior, en el presente estudio se evaluaron seis materiales criollos de México de L. siceraria en la producción de dos variedades de sandía, con el objetivo de seleccionar materiales promisorios para la producción y calidad de sandía.

\section{Materiales y Métodos}

El experimento se realizó en el Campo Experimental del Instituto de Ciencias Agrícolas de la Universidad Autónoma de Baja California, México $\left(32.40^{\circ} \mathrm{N}\right.$ y $\left.115.2^{\circ} \mathrm{O}\right)$ en el ciclo primaveraverano 2015. Variedades diploides de sandía 2800 y Sangría fueron injertadas sobre seis portainjertos criollos de L. siceraria colectados en México (Tabla 1). La técnica de injerto empleada fue de púa descrita por Maroto et al. (2002). Una vez realizado el injerto, las plántulas se mantuvieron en cámara húmeda ( 80 a $90 \%$ de humedad) con temperatura de 20 a $25^{\circ} \mathrm{C}$ por un período de siete días, posteriormente se redujo gradualmente la humedad hasta un $60 \%$ en un lapso de cuatro días.

La plantación se llevó acabo a campo abierto en suelo de textura arcillosa con $\mathrm{pH}$ de 8. El trasplante se hizo cuando las plantas presentaron cuatro hojas verdaderas a un marco de plantación de $0,90 \mathrm{~m}$ x 3,75 m. El experimento se estableció

Tabla 1. Ubicación geográfica y tamaño de fruto de materiales criollos L. siceraria colectados en México.

\begin{tabular}{|c|c|c|c|c|c|}
\hline \multirow{2}{*}{ Clave } & \multirow{2}{*}{ Sitio de colecta } & \multicolumn{2}{|c|}{ Coordenadas geográficas } & \multirow{2}{*}{$\begin{array}{c}\text { Altitud } \\
\text { msnm }\end{array}$} & \multirow{2}{*}{$\begin{array}{l}\text { Tamaño de fruto } \\
(\mathrm{ml})\end{array}$} \\
\hline & & Latitud norte & $\overline{\text { Longitud oeste }}$ & & \\
\hline L43 & Ensenada, Baja California & $30^{\circ} 30^{\prime} 22,20^{\prime \prime}$ & $115^{\circ} 56^{\prime} 09,11^{\prime \prime}$ & 13 & $\begin{array}{c}2.591 \\
\text { (grande) }\end{array}$ \\
\hline L46 & Silacayoapan, Oaxaca & $17^{\circ} 30^{\prime} 08,47^{\prime \prime}$ & $98^{\circ} 08^{\prime} 18,40^{\prime \prime}$ & 1.661 & $\begin{array}{c}361 \\
\text { (pequeño) }\end{array}$ \\
\hline L48 & Ensenada, Baja California & $30^{\circ} 50^{\prime} 22,76^{\prime \prime}$ & $116^{\circ} 03^{\prime} 53,39^{\prime \prime}$ & 27 & $\begin{array}{c}2.342 \\
\text { (grande) }\end{array}$ \\
\hline L50 & Ensenada, Baja California & $30^{\circ} 03^{\prime} 20,48^{\prime \prime}$ & $116^{\circ} 03^{\prime} 53,57^{\prime \prime}$ & 26 & $\begin{array}{c}2.512 \\
\text { (grande) }\end{array}$ \\
\hline L54 & Río Verde, San Luis Potosí & $21^{\circ} 53^{\prime} 40,34^{\prime \prime}$ & $100^{\circ} 02^{\prime} 48,86^{\prime \prime}$ & 1.011 & $\begin{array}{c}2.117 \\
\text { (mediano) }\end{array}$ \\
\hline L56 & Cuautla, Morelos & $18^{\circ} 48^{\prime} 46,07^{\prime}$ & $98^{\circ} 57^{\prime} 55,40^{\prime \prime}$ & 1.289 & $\begin{array}{c}309 \\
\text { (pequeño) }\end{array}$ \\
\hline
\end{tabular}


en un arreglo factorial $2 \times 7$ dentro de un diseño completamente al azar, replicando cada tratamiento tres veces y una unidad experimental de 18 plantas. Los factores de estudio fueron variedad (2800 y Sangría) y combinación de injerto (control y portainjertos L43, L46, L48, L50, L54 y L56), los que generaron 14 tratamientos (Tabla 2).

En el manejo del cultivo se aplicaron los riegos por sistema de goteo, el volumen se estimó de acuerdo a la evapotranspiración del cultivo (Etc), la que fue estimada como el producto de la evaporatranspiración del cultivo de referencia (ETo) y el coeficiente del cultivo (Kc) (Allen et al., 2006). La fertilización fue 129-92-154 NPK complementada con $61.5 \mathrm{~kg} \mathrm{CaO}$ y $15 \mathrm{~kg} \mathrm{MgO}$ (Fernández, 1998). Se emplearon abejas (Apis mellifera) para asegurar una buena polinización, en una relación cuatro colmenas $\mathrm{ha}^{-1}$. En la cosecha de los frutos se utilizó como criterio el secado de la estipula y zarcillo próximo al pedúnculo del fruto.

Las variables evaluadas fueron producción precoz $(\%)$, rendimiento de fruto $\left(\mathrm{t} \mathrm{ha}^{-1}\right)$, firmeza de pulpa (Newton), porcentaje de pulpa (\%) y

Tabla 2. Relación de tratamientos empleados en la evaluación de dos variedades sandia sin injerto e injertadas en seis portainjertos de L. siceraria.

\begin{tabular}{cll}
\hline $\begin{array}{c}\text { Combinación } \\
\text { de injerto } \\
(\mathrm{CI})\end{array}$ & \multicolumn{2}{c}{ Variedad (V) } \\
\cline { 2 - 3 } Control & $\mathrm{T} 1=2800$ & \multicolumn{1}{c}{ Sangría } \\
L43 & $\mathrm{T} 2=2800 / \mathrm{L} 43$ & $\mathrm{~T}$ T9=Sangría/L43 \\
L46 & $\mathrm{T} 3=2800 / \mathrm{L} 46$ & $\mathrm{~T} 10=$ Sangría/L46 \\
L48 & $\mathrm{T} 4=2800 / \mathrm{L} 48$ & $\mathrm{~T} 11=$ Sangría/L48 \\
L50 & T5=2800/L50 & T12=Sangría/L50 \\
L54 & T6=2800/L54 & T13=Sangría/L54 \\
L56 & T7=2800/L56 & T14=Sangría/L56 \\
\hline
\end{tabular}

contenido de sólidos solubles ( $\left.{ }^{\circ} \mathrm{Bix}\right)$. La producción precoz se determinó como la razón del rendimiento acumulado de los dos primeros cortes entre el rendimiento total, expresado en porcentaje, mientras que, en rendimiento se consideró la sumatoria de cuatro cortes realizados. La calidad de fruto se determinó en tres frutos por repetición tomados al azar en el segundo corte y en cada uno de los frutos se realizaron tres mediciones de firmeza y ${ }^{\circ}$ Brix. La firmeza se cuantificó con un medidor de fuerza digital Chatillon DFE-100 (AMETEK Inc., Florida, USA), y ${ }^{\circ}$ Brix con un refractómetro Digital Reichert AR200 (Reichert Inc., New York, USA). El porcentaje de pulpa se determinó con respecto al peso total del fruto.

Los datos generados se analizaron con el programa estadístico SAS 9,1, mediante el procedimiento GLM (General Linear Models) y las medias se compararon con la prueba de DMS (Diferencia mínima significativa) a un nivel de significancia de 0,05 .

\section{Resultados y Discusión}

La precocidad en la producción de sandía por efecto de las variedades, se encontró que la variedad 2800 superó en $12.35 \%$ en producción a Sangría en los dos primeros cortes (Tabla 3 y 4), mientras que al comparar el efecto de los portainjertos, la producción precoz se incrementó en $15,76 \%$ con respecto a la plantas sin injerto, sobresaliendo las combinaciones con el portainjerto L46, donde los incrementos fueron hasta del $19,22 \%$. La precocidad estuvo asociada a la aparición prematura de flores pistiladas (datos no mostrados), como lo reportó Yamasaki et al. (1994). Contrariamente, en otros estudios, se ha reportado menor precocidad en cosecha de sandía injertada en calabaza, lo que se atribuye

Tabla 3. Análisis de varianza de producción y calidad de frutos de sandía en dos variedades de sandía sin injerto e injertadas en seis portainjertos de L. siceraria.

\begin{tabular}{cccccc}
\hline $\begin{array}{c}\text { Fuente de } \\
\text { varianza }\end{array}$ & $\begin{array}{c}\text { Producción } \\
\text { precoz } \\
(\%)\end{array}$ & $\begin{array}{c}\text { Rendimiento } \\
\text { total } \\
\left(\mathrm{t} \mathrm{ha}^{-1}\right)\end{array}$ & $\begin{array}{c}\text { Firmeza de } \\
\text { pulpa } \\
(\mathrm{N})\end{array}$ & $\begin{array}{c}\text { Porcentaje } \\
\text { de pulpa } \\
(\%)\end{array}$ & $\begin{array}{c}\text { Contenido de sólidos } \\
\text { solubles } \\
\left({ }^{\circ} \text { Brix }\right)\end{array}$ \\
\hline $\mathrm{V}$ & $* *$ & $\mathrm{~ns}$ & $\mathrm{~ns}$ & $* *$ & $* * *$ \\
$\mathrm{CI}$ & $*$ & $*$ & $* * *$ & $\mathrm{~ns}$ & $* * *$ \\
$\mathrm{~V} * \mathrm{CI}$ & $\mathrm{ns}$ & $\mathrm{ns}$ & $\mathrm{ns}$ & $\mathrm{ns}$ & $* *$ \\
\hline
\end{tabular}

ns no significativo, $*$ nivel de significancia al $0,05, * *$ nivel de significancia al $0,01, * * *$ nivel de significancia al 0,001 . $\mathrm{V}=$ variedad y $\mathrm{CI}=$ combinación de injerto. 
Tabla 4. Comparación de medias de las variables producción precoz y rendimiento total de sandía en dos variedades sin injerto e injertadas en seis portainjertos de L. siceraria.

\begin{tabular}{cccccccc}
\hline \multirow{2}{*}{ Combinación injerto } & \multicolumn{3}{c}{ Producción precoz $(\%)$} & & \multicolumn{3}{c}{ Rendimiento total (t ha $\left.{ }^{-1}\right)$} \\
\cline { 2 - 3 } & 2800 & Sangría & Media & & 2800 & Sangría & Media \\
\hline Control & 68,88 & 61,54 & $65,21^{\mathrm{B}}$ & & 31,98 & 29,24 & $30,61^{\mathrm{B}}$ \\
L43 & 90,44 & 74,21 & $82,32^{\mathrm{A}}$ & & 58,44 & 49,47 & $53,95^{\mathrm{A}}$ \\
L46 & 83,87 & 84,99 & $84,43^{\mathrm{A}}$ & & 59,42 & 34,06 & $46,74^{\mathrm{A}}$ \\
L48 & 87,95 & 74,27 & $81,11^{\mathrm{A}}$ & & 54,49 & 49,05 & $51,77^{\mathrm{A}}$ \\
L50 & 85,02 & 77,41 & $81,21^{\mathrm{A}}$ & 53,65 & 51,70 & $52,68^{\mathrm{A}}$ \\
L54 & 91,46 & 63,34 & $77,40^{\mathrm{A}}$ & & 48,96 & 42,03 & $45,49^{\mathrm{AB}}$ \\
L56 & 86,76 & 72,13 & $79,44^{\mathrm{A}}$ & & 53,74 & 52,16 & $52,95^{\mathrm{A}}$ \\
Media & $84,91^{\mathrm{a}}$ & $72,56^{\mathrm{b}}$ & & 51,52 & 43,96 & \\
\hline
\end{tabular}

$\mathrm{AB}$ Medias con diferente letra en la misma columna son estadísticamente diferentes $(\mathrm{P}<0,05)$.

ab Medias con diferente letra en la misma fila son estadísticamente diferentes $(\mathrm{P}<0,05)$.

a mayor absorción de nitrógeno expresado en mayor crecimiento vigoroso de la plantas y retraso en la maduración de los frutos (Huang et al., 2016). Estos mismos investigadores señalan que Lagenaria como portainjerto son menos eficientes en la absorción de nutrientes de $\mathrm{N}, \mathrm{K}$ y $\mathrm{Mg}$, lo que explicaría la respuesta de mayor precocidad registrada en el presente estudio.

En rendimiento de fruto (Tablas 3 y 4), se encontró que la mayoría de los portainjertos empleados en ambas variedades, incrementaron en $20 \%$ el rendimiento en comparación con las plantas sin injerto. Este efecto inducido por L. siceraria, también ha sido reportado por Yetisir y Sari (2003) y Karaca et al. (2012), quienes coinciden en señalar aumentos en rendimiento que oscila de $27 \%$ a $107 \%$, producto de mayor número y tamaño de los frutos. Esta superioridad en la producción se atribuye a mayores tasas de absorción de agua y nutrientes por medio de las raíces, así como la asimilación de $\mathrm{CO}_{2}$ por las hojas (Rouphael et al., 2008). Otro factor involucrado es mayor proporción de flores femeninas (Islam et al., 2013).

La firmeza de la pulpa de los frutos es un atributo relacionado con varios factores anatómicos que pueden ser alterados por el injerto. En nuestros resultados se encontró una reducción de 3,9 N por efecto del injerto independientemente de la variedad, con excepción de los portainjertos L46 y L56 que tuvieron frutos con firmeza similar a los cosechados en plantas sin injerto (Tablas 3 y 5). En estudios similares con portainjertos de calabaza, la firmeza registrada ha sido superior con respecto a las plantas sin injerto, atribuyéndose a contenidos altos de $\alpha$ celulosa (Shinbori et al., 1981). En este sentido, es probable que los frutos cosechados en plantas injertadas en $L$. siceraria contengan menor contenido de celulosa. Diferencias en la firmeza de los frutos, también están dadas por variación en los estados de turgencia de las células, generándose menor resistencia de la pared celular en células turgentes (Kader, 2002) $\mathrm{y}$ al respecto, se ha documentado que los injertos inducen mayores tasas de absorción de agua (Rouphael et al., 2008), lo que podría relacionarse con la menor firmeza registrada.

El porcentaje de pulpa en los frutos (Tablas 3 y 5), fue diferente entre las variedades empleadas, registrándose 4,58\% más de pulpa en la variedad 2800 con respecto a Sangría. En cuanto al efecto de los portainjertos, no se tuvieron cambios en la cantidad de pulpa, lo que coincide con Proietti y colaboradores (2008), quienes reportaron valores similares de porcentaje de pulpa en frutos provenientes de plantas de sandía minivariedad "Ingrid" en condición normal e injertada. Otros resultados también confirman que en melón, los portainjertos no modifican la proporción de corteza, pulpa y semilla en fruto (Colla et al., 2010).

El contenido de sólidos solubles ( $\left.{ }^{\circ} \mathrm{Brix}\right)$ en los frutos de sandía (Tablas 3 y 6), se encontró que la variedad Sangría interacciona positivamente con los portainjertos, observándose incrementos de 1,01 a 2,60 ${ }^{\circ}$ Brix con respecto al testigo. En caso contrario, la variedad 2800 no modificó este parámetro al combinarse con los diferentes portainjertos, a excepción de L56, que registró estadísticamente menor concentración. Estos 
Tabla 5. Comparación de medias de las variables firmeza y porcentaje de pulpa en frutos de sandía de dos variedades sin injerto e injertadas en seis portainjertos de L. siceraria.

\begin{tabular}{|c|c|c|c|c|c|c|}
\hline \multirow{2}{*}{ Combinación injerto } & \multicolumn{3}{|c|}{ Firmeza de pulpa $(\mathrm{N})$} & \multicolumn{3}{|c|}{ Porcentaje de pulpa (\%) } \\
\hline & V. 2800 & V. Sangría & Media & 2800 & Sangría & Media \\
\hline Control & 15,77 & 15,58 & $15,67^{\mathrm{A}}$ & 75,70 & 66,05 & 70,88 \\
\hline L43 & 10,88 & 11,78 & $11,33^{\mathrm{DE}}$ & 75,70 & 74,81 & 75,26 \\
\hline L46 & 14,73 & 12,63 & $13,68^{\mathrm{ABC}}$ & 82,44 & 68,33 & 75,39 \\
\hline L48 & 10,33 & 10,66 & $10,49^{\mathrm{E}}$ & 71,63 & 69,39 & 70,51 \\
\hline L50 & 12,55 & 14,25 & $13,40^{\mathrm{BCD}}$ & 75,93 & 75,84 & 75,89 \\
\hline L54 & 11,78 & 11,74 & $11,76^{\mathrm{CDE}}$ & 72,45 & 71,27 & 71,86 \\
\hline L56 & 14,69 & 14,95 & $14,82^{\mathrm{AB}}$ & 74,83 & 70,96 & 72,90 \\
\hline Media & 12,96 & 13,08 & & $75,53^{\mathrm{a}}$ & $70,95^{\mathrm{b}}$ & \\
\hline
\end{tabular}

AB Medias con diferente letra en la misma columna son estadísticamente diferentes $(\mathrm{P}<0,05)$.

ab Medias con diferente letra en la misma fila son estadísticamente diferentes $(\mathrm{P}<0,05)$.

Tabla 6. Comparación de medias de la variable ${ }^{\circ}$ Brix en frutos de sandía de dos variedades sin injerto e injertadas en seis portainjertos de L. siceraria.

\begin{tabular}{cccr}
\hline \multirow{2}{*}{$\begin{array}{c}\text { Combinación } \\
\text { injerto }\end{array}$} & $\begin{array}{c}c \\
2800 \\
\left({ }^{\circ} \text { Brix }\right)\end{array}$ & $\begin{array}{c}\text { Sangría } \\
\left({ }^{\circ} \text { Brix }\right)\end{array}$ & Media \\
\hline Control & $10,22^{\mathrm{AB}}$ & $9,14^{\mathrm{C}}$ & 9,68 \\
L43 & $10,16^{\mathrm{AB}}$ & $11,12^{\mathrm{AB}}$ & 10,64 \\
L46 & $9,45^{\mathrm{B}}$ & $11,41^{\mathrm{A}}$ & 10,43 \\
L48 & $10,53^{\mathrm{A}}$ & $11,27^{\mathrm{A}}$ & 10,90 \\
L50 & $10,22^{\mathrm{AB}}$ & $10,82^{\mathrm{AB}}$ & 10,52 \\
L54 & $10,42^{\mathrm{A}}$ & $11,74^{\mathrm{A}}$ & 11,08 \\
L56 & $9,32^{\mathrm{C}}$ & $10,15^{\mathrm{BC}}$ & 9,73 \\
Media & 10,05 & 10,81 & \\
\hline
\end{tabular}

ABC Medias con diferente letra dentro de variedad son estadísticamente diferentes $(\mathrm{P}<0,05)$.

resultados demuestran que L. siceraria como portainjerto, mantiene o incrementa el contenido de ${ }^{\circ}$ Brix según la variedad de sandía, como fue reportado por Karaca et al. (2012).

\section{Conclusiones}

Portainjertos criollos de L. siceraria inducen mayor precocidad y rendimiento con respecto a la planta sin injerto. La relación pulpa-peso total del fruto es una característica que no fue alterada por los portainjertos de Lagenaria; sin embargo, esta especie puede disminuir la firmeza de la pulpa. El contenido de ${ }^{\circ}$ Brix en el fruto es un parámetro que se favorece con el injerto, aunque el nivel de respuesta depende de la combinación variedad/ portainjerto. Los portainjertos con mayor efecto en precocidad, rendimiento y calidad de fruto fueron L43 y L50, los que pueden ser considerados para programas de mejoramiento posteriores.

\section{Agradecimientos}

Los autores agradecen a la Universidad Autónoma de Baja California, por el apoyo otorgado por medio de la $17^{\mathrm{a}}$ Convocatoria Interna de Proyectos de Investigación.

\section{Literatura Citada}

Alan, O.; Ozdemir, N.; Gunen, Y.

2007. Effect of grafting on watermelon plant growth, yield and quality. Journal of Agronomy, 6 (2): 362-365.

Allen, G.; Pereira, S.; Raes, D.; Smith, M.

2006. Evapotranspiración del cultivo: Guías para la determinación de los requerimientos de agua de los cultivos. FAO. Riego y Drenaje. Roma, Italia. 299 p.

Barrett, E.; Zhao, X.; McSorley, R.

2012. Grafting for root-knot nematode control and yield improvement in organic heirloom tomato production. Hortscience, 47 (5): 614-620.
Chimonyo, P.; Modi, A.

2013. Seed Performance of Selected Bottle Gourd (Lagenaria siceraria (Molina) Stand1.). American Journal of Experimental Agriculture, 3 (4): 740-766.

Colla, G.; Cardona, M.; Cardarelli, M.; Rouphael, Y. 2010. Improving nitrogen use efficiency in melon by grafting. Hortscience, 45 (4): 559-565.

Colla, G.; Roupahel, Y.; Cardarelli, M.; Rea, E.

2006. Effect of salinity on yield, fruit quality, leaf gas exchange, and mineral composition of grafted watermelon plants. Hortscience, 41 (3): 622-627. 
Crino, P.; Bianco, L.; Rouphael, Y.; Colla, G.; Saccardo, F.; Paratore, A.

2007. Evaluation of rootstock resistance to fusarium wilt and gummy stem blight and effect on yield and quality of a grafted 'inodorus' melon. Hortscience, 42 (3): 521-525.

Fernández, C.

1998. Técnicas de producción de frutas y hortalizas en los cultivos protegidos del Sureste español. Ed. Caja rural de Almería. Almería, España. 445 p.

Huang, Y.; Zhao L.; Kong, Q.; Cheng, F.; Niu, M.; Xie, J.;

Muhammad, N.; Bie, Z.

2016. Comprehensive mineral nutrition analysis of watermelon grafted onto two different rootstocks. Horticultural Plant Journal, 2 (2): 105-113.

Islam, S.; Bashar, K.; Howlader, A.; Sarker, U.; Al-Mamun, H. 2013. Effect of grafting on watermelon growth and yield. Khon Kaen Agriculture Journal, 41: 284-289.

Kader, A.

2002. Fruits in the global market. In M. Knee (Ed.) Fruit quality and its biological basis. USA. pp. 1-6.

Karaca, F.; Yetisir, H.; Solmaz, I.; Candir, E.; Kurt, S.; Sari, N.; Guler, Z.

2012. Rootstock potential of Turkish Lagenaria siceraria germplasm for watermelon: plant growth, yield and quality. Turkish Journal of Agriculture and Forestry, 36: 167-177.

Lee, M.; Kubota, C.; Tsao, J.; Bie, Z.; Hoyos, P.; Morra, L.; Oda, M. 2010. Current status of vegetable grafting: Diffusion, grafting techniques, automation. Scientia Horticulturae Rewiews, 127: 93-105.

Lira, R.; Rodríguez, I.

2006. Catálogo de la familia Cucurbitaceae de México. Unidad de Biología, Tecnología y Prototipos, FES Iztacala, Base de datos SNIB-Conabio DS002.

Maroto, V.; Borrego, I.; Miguel, A.; Pomares, F. 2002. El cultivo de la sandía. Ed. Mundi-Prensa. México. 322 p. Petropoulos, A.; Olympios, C.; Ropokis, A.; Vlachou, G.; Ntatsi, G.; Paraskevopoulos, A.; Passam, C.

2014. Fruit volatiles, quality, and yield of watermelon as affected by grafting. Journal of Agricultural Science and Technology, 16: 873-885.
Proietti, S.; Rouphael, Y.; Colla, G.; Cardarelli, M.; De Agazio, M.; Zacchini, M.; Rea, E.; Moscatello, S.; Battistelli, A.

2008. Fruit quality of mini-watermelon as affected by grafting and irrigation regimes. Journal of the Science of Food and Agriculture, 88: 1107-1114.

Richardson, J.B.

1972. The pre-columbian distribution of the bottle gourd (Lagenaria siceraria): a re-evaluation. Economic Botany, 26: 265-273.

Rouphael, Y.; Cardarelli, M.; Colla, G.; Rea, E. 2008. Yield, mineral composition, water relations, and water use efficiency of grafted mini-watermelon plants under deficit irrigation. Hortscience, 43 (3): 730-736.

Schwarz, D.; Rouphael, Y.; Colla, G.; Venema, H.

2010. Grafting as a tool to improve tolerance of vegetables to abiotic stresses: Thermal stress, water stress and organic pollutants. Scientia Horticulturae, 127: 162-171.

Shinbori, F.; Kota, N.; Yoshino, A.

1981. Studies on the maturation physiology and the quality of watermelon. II. Influence of rootstocks on the growth properties and the quality in grafting culture. Bulletin of Chiba-Ken Agricultural Experiment Station, 22: 21-27.

Soteriou, A.; Kyriacou, M.; Siomos, S.; Gerasopoulos, D.

2014. Evolution of watermelon fruit physicochemical and phytochemical composition during ripening as affected by grafting. Food Chemistry, 165: 282-289.

Tarchoun, N.; Boughalleb, N.; EL Mbarki, A.

2005. Agronomic evaluation of nine cucurbit rootstocks and watermelon grafted (Citrullus lanatus T.). Revue de L'INAT, 20 (2): 125-140.

Yamasaki, A.; Yamashita, M.; Furuya, S.

1994. Mineral concentrations and cytokinin activity in the xylem exudate of grafted watermelon as affected by rootstocks and crop load. Journal Japanese Society for Horticultural Science, 62 (4): 817-826.

Yetisir, H.; Sari, N.

2003. Effect of different rootstock on plant growth, yield and quality of watermelon. Australian Journal of Experimental Agriculture, 43: 1269-1274. 